\title{
Seed Germination and Seedling Growth Responses to Different Sources and Application Rates of Hydrothermal Carbonization Processed Liquid
}

\author{
Yuhang $\mathrm{He}^{1}$, Quan $\mathrm{He}^{2}$, Kris Pruski ${ }^{1}$, Bishnu Acharya ${ }^{3,4}$ \& Lord Abbey ${ }^{1}$ \\ ${ }^{1}$ Department of Plant, Food, and Environmental Sciences, Faculty of Agriculture, Dalhousie University, 50 \\ Pictou Road, Bible Hill, NS B2N 5E3, Canada \\ ${ }^{2}$ Department of Engineering, Faculty of Agriculture, Dalhousie University, 39 Cox Road, Bible Hill, NS B2N \\ 5E3, Canada \\ ${ }^{3}$ Faculty of Sustainable Design Engineering, University of Prince Edward Island, 550 University Avenue, \\ Charlottetown, PE C1A 4P3, Canada \\ ${ }^{4}$ Department of Chemical and Biological Engineering, University of Saskatchewan, 05 Administration Place, \\ Saskatoon, SK S7N 5A2, Canada \\ Correspondence: Lord Abbey, Department of Plant, Food, and Environmental Sciences, Faculty of Agriculture, \\ Dalhousie University, 50 Pictou Road, Bible Hill, NS B2N 5E3, Canada. E-mail: loab07@gmail.com. ORCID: \\ 0000-0003-2219-1752
}

Received: August 24, 2021 Accepted: September 30, 2021 Online Published: October 10, 2021

doi:10.5539/sar.v10n4p1 URL: https://doi.org/10.5539/sar.v10n4p1

\begin{abstract}
Hydrothermal carbonization processed liquid (HTCPL) is a by-product of hydrothermal carbonization of biomass, which is used sparingly as natural fertilizer. A study was performed in the Faculty of Agriculture, Dalhousie University (Canada) between June 2019 and April 2020 to evaluate the elemental composition of HTCPL derived from three different biomass feedstock; namely, seafood compost; buckwheat (Fagopyrum esculentum), and willow (Salix babylonica). Different HTCPL application rates (0-10\%) were tested on seed germination and seedling growth of pea (Pisum sativum), sunflower (Helianthus annuus), pac choi (Brassica rapa subsp. chinensis), kale (Brassica oleracea var. sabellica) and lettuce (Lactuca sativa). Elemental composition was higher in the HTCPLs compared to their respective feedstocks except for nitrogen. The 5\% and $10 \%$ willow HTCPL with a pH between 3.8-4.0 inhibited seed germination and seedling growth compared to the other treatments with a $\mathrm{pH}$ range between 4.6-5.8. Kale, lettuce and sunflower radicle and hypocotyl growth were promoted following treatments of their respective seeds with seafood compost HTCPL while pea radicle and hypocotyl lengths were best promoted by 5\% buckwheat and 10\% seafood compost HTCPLs. Comparatively, $0.5 \%$ willow HTCPL increased surface area of seedling radicles while $1 \%$ willow and $0.5 \%$ buckwheat HTCPLs increased surface area of hypocotyls, irrespective of plant species. The distinction among the treatments was demonstrated on a 2-dimensional principal component analysis biplot that explained $89 \%$ of the variations in dataset. Overall, buckwheat HTCPL proved to be more effective at increasing seed germination and seedling growth compared to the other HTCPLs. The inhibitory effect of willow HTCPL at high application rate (5-10\%) were obvious for all plant species. A comprehensive non-targeted chemical profile of HTCPL will help to explain mechanisms.
\end{abstract}

Keywords: biomass conversion, biostimulant, nutrient solution, seed germination, seedling establishment

\section{Introduction}

Hydrothermal carbonization (HTC) is a thermochemical process used to convert organic materials at temperatures between $180^{\circ}$ and $350^{\circ} \mathrm{C}$ to value-added products (Saetea and Tippayawong, 2013). Biomass such as plants, composts, and sewage sludge are commonly used as feedstocks for HTC (Saetea and Tippayawong, 2013; Funke, 2015; Yao et al., 2016; Idowu et al., 2017). Typically, HTC products comprised of solid, liquid and a small gas fraction. The most valuable HTC product is solid hydrochar, which has many uses including soil amendment, water purifier, and metal adsorbent. The liquid product of HTC is referred to as hydrothermal carbonization processed liquid (HTCPL), which can potentially be used as a biostimulant or nutrient solution in 
agriculture (Sun, Sumida, \& Yoshikawa, 2014; Funke, 2015; Yao et al., 2016), but it has not been fully studied yet.

The type and particle size of feedstock, temperature, heating rate, and residence time during the HTC process affect the yield and chemical composition of HTCPL (Saetea and Tippayawong, 2013; Funke, 2015; Idowu et al., 2017). For instance, Sun et al. (2014) extracted HTCPL from sewage sludge at three different temperatures (i.e., $180^{\circ} \mathrm{C}, 200^{\circ} \mathrm{C}$ and $220^{\circ} \mathrm{C}$ ), and diluted the extracted solution to cultivate komatsuna (Brassica rapa var. perviridis). They reported variations in chemical compositions of the HTCPLs, and promotion of komatsuna plant growth, especially with application of HTCPL extracted at $200^{\circ} \mathrm{C}$. Furthermore, HTCPL extracted from rosemary (Rosmarinus officinalis) showed significant antioxidant activity due to the presence of IS- $\alpha$-pinene and eucalyptol in the starting feedstock (Ma et al., 2013).

Although some studies suggested that HTCPL can replace synthetic chemical fertilizers (Sun et al., 2014), other studies did not fully support this view (Idowu et al. 2017). This is because the storage form of plant nutrients in the feedstock after the HTC process are not clear (Heilmann et al., 2010; Escala, Zumbuhl, Koller, Junge, \& Krebs, 2012; Funke, Mumme, Koon, \& Diakité, 2013; Reza, Lynam, Uddin, \& Coronella, 2013; Saetea and Tippayawong, 2013; Funke, 2015). Researchers reported that nitrogen (N) is the main element in HTCPL with more than 50\% in its organic form (Saetea and Tippayawong, 2013; Sun et al., 2014). On the other hand, phosphorus (P) was found to be locked in the solid component i.e., hydrochar (Escala et al., 2012; Heidari, Salaudeen, Dutta, \& Acharya, 2018) while other researchers suggested that P is mainly recovered in HTCPL (Heilmann et al., 2010; Reza et al., 2013; Saetea and Tippayawong, 2013). According to Idowu et al. (2017), large proportion of $\mathrm{N}$ was recovered in food waste hydrochar while potassium (K) was recovered in HTCPL, but the fate of $\mathrm{P}$ was not clear. These differences can be attributed to differences in feedstock and the conditions set for the HTC process in the various studies. Despite these contradictions, we hypothesized that most nutrients can be recovered in HTCPL using the appropriate feedstock and HTC conditions. So far, there are not many literature information on the effects of HTCPL on plant seed germination, and scholars have not formed a mainstream unified view. It is generally believed that the effect of HTCPL on seed germination is related to the composition and properties of feedstock (Bargmann, Rillig, Buss, Kruse, \& Kuecke, 2013; Saetea and Tippayawong, 2013; Funke, 2015). One study used HTCPL extracted from six different types of biomasses (i.e., grass, wood, straw, biogas digestate, and horse manure) to test their effects on the germination of Spring barely (Hordeum vulgare) (Bargmann et al., 2013). They proposed that undiluted HTCPL has an inhibitory effect on seed germination, and the main reason is related to the acidic components in HTCPL.

Germination rate is the most direct parameter to evaluate the viability of seeds. It is not sufficient to use only seed germination rate to determine seed germination activity (Yang et al., 2015). Germination index is a good parameter to describe the relationships between the germination rate and germination speed (Yang et al., 2015). Furthermore, germination potential provides an evaluation of the field performance of seeds (Yang et al., 2015). Moreover, seed vigor index reflects the sum of seed characteristics that determine the activity level and performance of seed germination and seed emergence ((Yousefi, Kartoolinejad, Bahmani, \& Naghdi, 2017). The length and surface area of plants are important indicators for assessing the growth of seedlings. In the immature seedling stage of plants, the length of plant radicle and hypocotyl is positively correlated with plant growth (Yang et al., 2015); while surface area of seedling is closely related to the absorption of water and nutrients of the immature plant (Yousefi et al., 2017).

Agriculture or forestry wastes are the main feedstocks for HTC. In the present study, we extracted HTCPLs from willow (Salix babylonica) softwood, seafood compost, and buckwheat (Fagopyrum esculentum) green foliage and tested them separately on different seeds. Willow contains acetic acid, carboxylic acid and indole acetic acid (Hagner et al., 2020). Seafood compost is rich in humic and non-humic substances including macro- and micro-elements and amino acids among others (Abbey, Annan, Asiedu, Esan, \& Iheshiulo, 2018). Buckwheat is a common rotation crop due to its high mineral nutrients, especially N and P (Salehi, Mehdi, Fallah, Kaul, \& Neugschwandtner, 2018). It is postulated that beneficial elements and bioactive compounds in willow, seafood compost and buckwheat may be retained in their respective HTCPLs to benefit plant growth and development. Moreover, there is little information on the application of willow, seafood compost and buckwheat HTCPLs on seed germination and seedling growth. Therefore, the objective of this study was to determine the effect of different application rates and sources of HTCPLs on seed germination, radicle (embryonic root) and hypocotyl (embryonic shoot) growth of several species. Pea (Pisum sativum), sunflower (Helianthus annuus), pac choi (Brassica rapa subsp. chinensis), kale (Brassica oleracea var. sabellica), and lettuce (Lactuca sativa) were the chosen species because they are commonly grown and consumed as microgreens for their nutrients and health benefits. 


\section{Materials and Methods}

Seed gemination and seedling growth experiments were performed in the Faculty of Agriculture, Dalhousie University (Canada) from June 2019 to April 2020. Two separate experiments were carried out to assess the impact of high (5-10\%) or low (2: 0-2.5\%) application rates of willow, seafood compost and buckwheat HTCPLs on seed germination and seedling growth components. The HTCPL extraction was performed in the Faculty of Sustainable Design Engineering, University of Prince Edward Island (Canada) in May 2019.

\subsection{Plant Materials}

Seafood compost (Greenhouse Gold ${ }^{\mathrm{TM}}$, NB, Canada) was purchased from a local retailer in Truro. Willow tree softwood branches and buckwheat green foliage were obtained from UPEI experimental field and Dalhousie University demonstration garden, respectively. Organically certified seeds of kale, pac choi, pea, sunflower and lettuce were purchased from West Coast Seed, BC, Canada. CYG ${ }^{\mathrm{TM}}$ seed germination pouches were purchased from Mega International, MN, USA.

\subsection{HTCPL Extraction}

Fifty (50) g of ground feedstock (i.e., willow softwood, seafood compost and buckwheat green foliage) was separately added to $450 \mathrm{~g}$ of distilled water in a Parr 4848 pressure reactor (Parr Instrument Co., IL, USA). The air in the reactor was purged with $\mathrm{N}$ gas to prevent oxidation during reaction. The reactor was set at $200^{\circ} \mathrm{C}$ and $100 \mathrm{psi}$ at an average heating rate of $4.9^{\circ} \mathrm{C} / \mathrm{min}$ and a residence time of $60 \mathrm{~min}$. Finally, the reactor was cooled down to room temperature (i.e., $c a .22^{\circ} \mathrm{C}$ ), and a filter paper was used to separate the solid hydrochar to obtain the HTCPL.

\subsection{Analysis of Feedstocks and HTCPLs}

A thirty (30) g sample of each ground feedstock and $10 \mathrm{ml}$ of each HTCPL were sent to Prince Edward Island Analytical Laboratories, PEI, Canada for elemental analysis. Chemical elements were analyzed using 5800 inductively coupled plasma-mass spectrometry/inductively coupled plasma optical emission spectroscopy (Agilent Technologies, CA, USA) according to EPA method 200.8/EPA 200.7 (Standard Operation Procedures \#4.M01/4.M29). Each sample was analyzed calorimetrically for total N concentration (Cataldo, Schrader, \& Youngs, 1974). The $\mathrm{pH}$ of each HTCPL was measured using a multi-parameter water quality meter (Oakton Instruments, IL, USA) in the Compost and Biostimulant Laboratory, Dalhousie University Faculty of Agriculture.

\section{Experiment 1: High Application Rate of HTCPL}

The first experiment was performed to assess the effects of high rates ( 0 - distilled water, $5 \%$ and $10 \%)$ of willow, seafood compost and buckwheat HTCPLs on seed germination and seedling growth components of kale, pac choi, pea, sunflower and lettuce. Seeds were placed in the troughs of $\mathrm{CYG}^{\mathrm{TM}}$ seed germination pouches $(36 \mathrm{~cm}$ length, $16.5 \mathrm{~cm}$ width and $3 \mathrm{~cm}$ depth) followed by addition of $20 \mathrm{ml}$ of HTCPL per treatment. $5 \mathrm{~mL}$ of the respective HTCPL was added every other day until the experiment was terminated. The pouched seeds were placed in the dark at room temperature $\left(c a .22^{\circ} \mathrm{C}\right)$. Protrusion of $0.5-\mathrm{mm}$ length of emerged radicle from a seed was recorded as germination. The experiment was terminated when the number of germinated seeds did not increase for three consecutive days.

\section{Experiment 2: Low Application Rate of HTCPL}

It was found in Experiment 1 that small seeds were the most sensitive and as such, kale was selected for the second experiment. Also, buckwheat and willow were selected for Experiment 2 because the former had the highest overall concentration of chemical elements, and the latter had the lowest. The goal of the second experiment was to assess the effects of low rates $(0 \%, 0.5 \%, 1 \%$ and $2.5 \%)$ of willow and buckwheat HTCPL on kale seed germination and seedling growth components. The kale seeds $(n=20)$ were treated separately with the HTCPLs as described in Experiment 1.

\subsection{Data Collection}

Experiments 1 and 2 were respectively arranged in a 3-factor and 2-factor randomized complete block design with three replications. Data $(\mathrm{n}=20)$ on seed germination rate, germination potential, germination index, and seed vigor index were recorded daily until the experiment was terminated after 10 to 12 days after seed treatment. Calculations of seed vigor and seed germination indices were based on descriptions by Yang et al. (2015) and Yousefi et al. (2017) as follows:

$$
\text { Germination rate }(\%)=\frac{\text { sum of germinated seeds }}{\text { total number of seeds }} \times 100
$$


Germination potential $(\%)=\frac{\text { sum of germinated seeds at peak germination }}{\text { total number of seeds }} \times 100$

Germination index $=\sum\left(G_{t} \div D_{t}\right) ; G_{t}$, number of seeds germinated on a given day and $D_{t}$, corresponding number of days of germination.

Seed vigor index $=$ germination rate $\times$ mean $\frac{S_{i}+R_{i}}{100} ; S_{i}$, shoot length and $R_{i}$, root length.

Germination index is used to describe relationship between seed germination rate and seed germination speed, and seed vigor index reflects the activity level and performance of seed germination and seedling emergence. Digital images of the seedlings, and lengths and surface areas of germinated seed hypocotyls and radicles were determined using STD4800 WinRHIZO root scanner (Epson, CA, USA).

\subsection{Statistical Analysis}

Data collected were subjected to a 3-way (Experiment 1) or 2-way (Experiment 2) analyses of variance (ANOVA) using Minitab version 19.1 (Minitab Inc., PA, USA). Tukey's method was used to separate treatment means when the ANOVA indicated significant difference between at least one treatment mean at $\mathrm{P} \leq 0.05$. Variance-covariance structure of the dataset was explained by two-dimensional principal component analysis (PCA) using Minitab version 18.1 (Minitab Inc., State College, PA, USA).

\section{Results}

\subsection{Feedstock and HTCPL Chemical Elements}

The different feedstocks (i.e., buckwheat green foliage, seafood compost and willow softwood) for the HTC had different concentrations of chemical elements. Comparatively, the buckwheat foliage had the highest macro-elements i.e., N, P, K, calcium (Ca) and magnesium (Mg); and micro-elements i.e., boron (B), copper ( $\mathrm{Cu})$ and zinc $(\mathrm{Zn})$ followed by the seafood compost and then the willow softwood (Table 1). P was not detected in willow softwood but was present in high concentration in the HTCPL. N was high in buckwheat HTCPL followed by seafood HTCPL and then willow HTCPL. In addition to $\mathrm{N}, \mathrm{P}, \mathrm{K}$ and $\mathrm{Mg}$ were remarkably high in buckwheat HCTPL followed by willow HCTPL and then seafood HTCPL (Table 1). Ca was very high in seafood HTCPL followed by willow HTCPL and then buckwheat HTCPL. Micro-elements i.e., boron (B), was high in willow HTCPL followed by buckwheat HTCPL and then seafood HTCPL. Copper $(\mathrm{Cu})$ contents of the three HTCPL were the same at $0.05 \mathrm{mg} / \mathrm{kg}$. Zinc (Zn) was reduced by 102 -fold and 271 -fold, respectively in the seafood HTCPL and buckwheat HTCPL compared to their respective feedstock before the HTC process. However, willow HTCPL retained $44 \%$ of the zinc $(\mathrm{Zn})$ content in the feedstock.

Table 1. Elemental composition of seafood compost, buckwheat foliage and willow softwood feedstocks and extracted hydrothermal carbonization processed liquids (HTCPL)

\begin{tabular}{lllllll}
\hline \multirow{2}{*}{ Elements } & \multicolumn{2}{l}{ Seafood compost $(\mathrm{mg} / \mathrm{kg})$} & \multicolumn{2}{l}{ Buckwheat foliage $(\mathrm{mg} / \mathrm{kg})$} & \multicolumn{2}{l}{ Willow softwood $(\mathrm{mg} / \mathrm{kg})$} \\
\cline { 2 - 7 } & Feedstock & HTCPL $^{1}$ & Feedstock & HTCPL & Feedstock & HTCPL \\
\hline Nitrogen & 7500 & 486 & 28900 & 1467 & 2600 & 163 \\
Phosphorus & 2610 & 3.5 & 5100 & 214.2 & $*$ ND & 68.6 \\
Potassium & 6520 & 12.85 & 28800 & 1574.5 & 400 & 231.25 \\
Calcium & 10750 & 199.6 & 11400 & 6.3 & 700 & 153.85 \\
Magnesium & 1800 & 24 & 6700 & 223.5 & 200 & 51.75 \\
Boron & 13.16 & 0.25 & 20.2 & 0.7 & 2.47 & 0.76 \\
Copper & 8.54 & 0.05 & 7.4 & 0.05 & 1.57 & 0.05 \\
Zinc & 30.6 & 0.3 & 54.2 & 0.2 & 13.9 & 6.15 \\
\hline
\end{tabular}

ND, element not detected.

The $\mathrm{pH}$ and alkalinity of willow HTCPL were lowest compared to those of seafood compost and buckwheat (Table 2). Comparatively, buckwheat HTCPL had intermediate $\mathrm{pH}$ of 4.6 but the highest alkalinity. Dilutions of the various HTCPLs raised their pH values (Table 3) to between 5.8 for 5\% SC HTCPL to 3.8 for $10 \%$ willow HTCPL (Table 3).

Table 2. Mean $\mathrm{pH}$ and alkalinity values of hydrothermal carbonization processed liquids (HTCPLs) obtained from SC (seafood compost), willow (Salix babylonica) branches, and buckwheat (Fagopyrum esculentum) green foliage at $180^{\circ} \mathrm{C}$ and $200^{\circ} \mathrm{C}$

\begin{tabular}{llll}
\hline & SC HTCPL & Buckwheat HTCPL & Willow HTCPL \\
\hline $\mathrm{pH}$ & 5.1 & 4.56 & 3.73 \\
Alkalinity $(\mathrm{mg} / \mathrm{kg})$ & 351.25 & 961.10 & 97.8 \\
\hline
\end{tabular}


Table 3. $\mathrm{pH}$ value of different concentrations of hydrothermal carbonization processed liquids (HTCPLs) extract from SC (seafood compost), buckwheat (Fagopyrum esculentum) green foliage and willow (Salix babylonica) branches

\begin{tabular}{lllllll}
\hline & $5 \%$ & $10 \%$ & $0.5 \%$ Buckwheat & $1 \%$ Buckwheat & 2.5\% Buckwheat & 5\% Buckwheat \\
& SC HTCPL & SC HTCPL & HTCPL & HTCPL & HTCPL & HTCPL \\
\hline $\mathrm{pH}$ & 5.75 & 5.21 & 5.51 & 5.37 & 5.01 & 4.66 \\
& $10 \%$ Buckwheat & $0.5 \%$ Willow & $1 \%$ Willow & $2.5 \%$ Willow & $5 \%$ Willow & $10 \%$ Willow \\
& HTCPL & HTCPL & HTCPL & HTCPL & HTCPL & HTCPL \\
$\mathrm{pH}$ & 4.58 & 4.87 & 4.58 & 4.33 & 4.04 & 3.75 \\
\hline
\end{tabular}

\section{Experiment 1: High Application Rate of HTCPL}

There were no significant $(\mathrm{P}>0.05)$ differences in the main effects (i.e., HTCPL type, HTCPL rate or plant species) and 2-way interactions. However, the 3-way interaction effect was significant $(\mathrm{P}<0.05)$. As such, data for the 3-way interaction were presented in Tables 2 and 3. Seafood compost HTCPL, especially at 5\% application rate significantly $(\mathrm{P}=0.001)$ increased seed germination rate (i.e., $87-100 \%)$ of all the plant species, and was not significantly $(\mathrm{P}<0.05)$ different from the control treatment (i.e., 92-100\%) (Table 4).

Table 4. Seed germination indices of kale (Brassica oleracea var. sabellica), lettuce (Lactuca sativa), pac choi (Brassica rapa subsp. chinensis), pea (Pisum sativum) and sunflower (Helianthus annuus) as affected by different sources and high rate of hydrothermal carbonization processed liquid (HTCPL) (different alphabetical letters indicate significant differences between the treatments using 3-way ANOVA and following Tukey's test at $\mathrm{P} \leq 0.05$, confidence intervals $=95 \%$ )

\begin{tabular}{|c|c|c|c|c|c|c|c|c|c|}
\hline \multirow[b]{2}{*}{ Plant } & \multirow[b]{2}{*}{ HTCPL treatment } & \multicolumn{2}{|c|}{ Germination rate } & \multicolumn{2}{|c|}{ Germination index } & \multicolumn{2}{|c|}{ Germination potential } & \multicolumn{2}{|c|}{ Seed vigor index } \\
\hline & & $(\%)$ & $\mathrm{sd}$ & 1 & $\mathrm{sd}$ & $(\%)$ & sd & 1 & sd \\
\hline Kale & Control & $98.3 \mathrm{a}$ & 0.0289 & $5.76 \mathrm{~cd}$ & 0.258 & $66.7 \mathrm{a}-\mathrm{g}$ & 0.0764 & $2.00 \mathrm{a}-\mathrm{c}$ & 0.1259 \\
\hline Kale & $5 \%$ Buckwheat & $93.3 \mathrm{a}$ & 0.0289 & $5.83 \mathrm{~cd}$ & 0.308 & $78.3 \mathrm{a}-\mathrm{e}$ & 0.00289 & $1.54 \mathrm{a}-\mathrm{f}$ & 0.393 \\
\hline Kale & $10 \%$ Buckwheat & $95.0 \mathrm{a}$ & 0.0866 & $5.83 \mathrm{~cd}$ & 0.269 & $73.3 \mathrm{a}-\mathrm{f}$ & 0.1258 & $1.37 \mathrm{~b}-\mathrm{g}$ & 0.356 \\
\hline Kale & $5 \%$ Seafood compost & $93.3 \mathrm{a}$ & 0.0577 & $5.59 \mathrm{c}-\mathrm{e}$ & 0.457 & $63.3 \mathrm{a}-\mathrm{g}$ & 0.1041 & $1.90 \mathrm{a}-\mathrm{g}$ & 0.1698 \\
\hline Kale & $10 \%$ Seafood compost & $93.3 \mathrm{a}$ & 0.0577 & $5.90 \mathrm{c}$ & 0.321 & $70.0 \mathrm{a}-\mathrm{g}$ & 0.1 & $1.53 \mathrm{a}-\mathrm{f}$ & 0.1539 \\
\hline Kale & $5 \%$ Willow & $48.3 \mathrm{c}$ & 0.179 & $1.94 \mathrm{i}-\mathrm{k}$ & 0.864 & $35.0 \mathrm{f}-\mathrm{j}$ & 0.1323 & $0.40 \mathrm{~h}-\mathrm{j}$ & 0.244 \\
\hline Kale & $10 \%$ Willow & $1.7 \mathrm{e}$ & 0.0289 & 0.041 & 0.0642 & $1.7 \mathrm{j}$ & 0.0289 & $0.30 \mathrm{j}$ & 0.00316 \\
\hline Lettuce & Control & $91.7 \mathrm{a}$ & 0.0289 & $7.55 \mathrm{~b}$ & 0.577 & $60.0 \mathrm{~b}-\mathrm{h}$ & 0.05 & $1.15 \mathrm{~b}-\mathrm{h}$ & 0.1678 \\
\hline Lettuce & $5 \%$ Buckwheat & $70.0 \mathrm{~b}$ & 0.0866 & $4.27 \mathrm{e}-\mathrm{g}$ & 0.959 & $40.0 \mathrm{~d}-\mathrm{j}$ & 0.18 & $0.68 \mathrm{~g}-\mathrm{j}$ & 0.238 \\
\hline Lettuce & $10 \%$ Buckwheat & $73.3 \mathrm{~b}$ & 0.0764 & $4.47 \mathrm{~d}-\mathrm{f}$ & 0.832 & $38.3 \mathrm{e}-\mathrm{j}$ & 0.0764 & $0.79 \mathrm{f}-\mathrm{j}$ & 0.1466 \\
\hline Lettuce & $5 \%$ Seafood compost & $91.7 \mathrm{a}$ & 0.0764 & $6.96 \mathrm{bc}$ & 0.705 & $48.3 \mathrm{c}-\mathrm{i}$ & 0.0764 & $1.54 \mathrm{c}-\mathrm{i}$ & 0.426 \\
\hline Lettuce & $10 \%$ Seafood compost & $86.7 \mathrm{ab}$ & 0.1528 & $5.81 \mathrm{~cd}$ & 1.126 & $30.0 \mathrm{~g}-\mathrm{j}$ & 0.05 & $1.11 \mathrm{~d}-\mathrm{h}$ & 0.251 \\
\hline Lettuce & $5 \%$ Willow & $46.7 \mathrm{c}$ & 0.0289 & $2.40 \mathrm{~h}-\mathrm{j}$ & 0.546 & $30.0 \mathrm{~g}-\mathrm{j}$ & 0.1323 & $0.32 \mathrm{~h}-\mathrm{j}$ & 0.186 \\
\hline Lettuce & $10 \%$ Willow & $28.3 \mathrm{~d}$ & 0.0289 & $1.08 \mathrm{j}-1$ & 0.1723 & $21.7 \mathrm{hij}$ & 0.0577 & $0.26 \mathrm{ij}$ & 0.1639 \\
\hline Pac choi & Control & $93.3 \mathrm{a}$ & 0.0289 & $7.78 \mathrm{ab}$ & 0.419 & $65.0 \mathrm{a}-\mathrm{g}$ & 0.173 & $1.03 \mathrm{a} \mathrm{ag}$ & 0.0942 \\
\hline Pac choi & $5 \%$ Buckwheat & $98.3 \mathrm{a}$ & 0.0289 & $9.08 \mathrm{a}$ & 0.52 & $76.7 \mathrm{a}-\mathrm{e}$ & 0.1041 & $1.08 \mathrm{~d}-\mathrm{i}$ & 0.1466 \\
\hline Pac choi & $10 \%$ Buckwheat & $96.7 \mathrm{a}$ & 0.0577 & $7.91 \mathrm{ab}$ & 0.684 & $81.7 \mathrm{a}-\mathrm{c}$ & 0.1443 & $0.64 \mathrm{~g}-\mathrm{j}$ & 0.197 \\
\hline Pac choi & $5 \%$ Seafood compost & $100.0 \mathrm{a}$ & 0 & $8.21 \mathrm{ab}$ & 0.609 & $85.0 \mathrm{a}-\mathrm{c}$ & 0.18 & 1.0 & 0.242 \\
\hline Pac choi & $10 \%$ Seafooc & $96.7 \mathrm{a}$ & 0.0289 & & 0.747 & $a-c$ & 607 & e-i & 0.0222 \\
\hline Pac choi & $5 \%$ Willow & $10.0 \mathrm{e}$ & 0.1 & $0.83 \mathrm{kl}$ & 0.833 & $10.0 \mathrm{ij}$ & 0.1 & $0.04 \mathrm{j}$ & 0.0347 \\
\hline Pac choi & $10 \%$ Willow & $0.2 \mathrm{e}$ & 0 & 0.061 & 0.0962 & $1.7 \mathrm{j}$ & 0.0289 & $0.03 \mathrm{j}$ & 0 \\
\hline Pea & Control & $97.5 \mathrm{a}$ & 0.05 & $2.75 \mathrm{hi}$ & 0.243 & $85.0 \mathrm{ab}$ & 0.1915 & $0.94 \mathrm{f}-\mathrm{i}$ & 0.384 \\
\hline Pea & $5 \%$ Buckwheat & $100.0 \mathrm{a}$ & 0 & $3.15 \mathrm{f}-\mathrm{i}$ & 0.0725 & $80.0 \mathrm{a}-\mathrm{c}$ & 0.0816 & $1.52 \mathrm{a}-\mathrm{f}$ & 0.0757 \\
\hline Pea & $10 \%$ Buckwheat & $100.0 \mathrm{a}$ & 0 & $2.80 \mathrm{hi}$ & 0.21 & $87.5 \mathrm{ab}$ & 0.1258 & $1.00 \mathrm{e}-\mathrm{i}$ & 0.1891 \\
\hline Pea & $5 \%$ Seafood compost & $97.5 \mathrm{a}$ & 0.05 & $3.02 \mathrm{~g}-\mathrm{i}$ & 0.1423 & $70.0 \mathrm{a}-\mathrm{f}$ & 0.0816 & $1.37 \mathrm{~b}-\mathrm{g}$ & 0.1335 \\
\hline Pea & $10 \%$ Seafood compost & $97.5 \mathrm{a}$ & 0.05 & $3.06 \mathrm{~g}-\mathrm{i}$ & 0.329 & $77.5 \mathrm{a}-\mathrm{d}$ & 0.206 & $1.42 \mathrm{~b}-\mathrm{g}$ & 0.297 \\
\hline Pea & $5 \%$ Willow & $95.0 \mathrm{a}$ & 0.0577 & $2.99 \mathrm{~g}-\mathrm{i}$ & 0.1892 & $75.0 \mathrm{a}-\mathrm{e}$ & 0.1915 & $0.88 \mathrm{f}-\mathrm{i}$ & 0.1774 \\
\hline Pea & $10 \%$ Willow & $100.0 \mathrm{a}$ & 0 & $2.95 \mathrm{~g}-\mathrm{i}$ & 0.104 & $82.5 \mathrm{a}-\mathrm{c}$ & 0.206 & $0.48 \mathrm{~h}-\mathrm{j}$ & 0.253 \\
\hline Sunflower & Control & $100.0 \mathrm{a}$ & 0 & $3.29 \mathrm{f}-\mathrm{h}$ & 0.0833 & $95.0 \mathrm{ab}$ & 0.1 & $1.5 \mathrm{a}-\mathrm{f}$ & 0.296 \\
\hline Sunflower & $5 \%$ Buckwheat & $100.0 \mathrm{a}$ & 0 & $3.33 \mathrm{f}-\mathrm{h}$ & 0 & $100.0 \mathrm{a}$ & 0 & $1.71 \mathrm{a}-\mathrm{e}$ & 0.068 \\
\hline Sunflower & $10 \%$ Buckwheat & $100.0 \mathrm{a}$ & 0 & $3.29 \mathrm{f}-\mathrm{h}$ & 0.0833 & $95.0 \mathrm{ab}$ & 0.1 & $1.33 \mathrm{c}-\mathrm{g}$ & 0.777 \\
\hline Sunflower & $5 \%$ Seafood compost & $100.0 \mathrm{a}$ & 0 & $3.29 \mathrm{f}-\mathrm{h}$ & 0.0481 & $95.0 \mathrm{ab}$ & 0.0577 & $2.09 \mathrm{ab}$ & 0.1557 \\
\hline Sunflower & $10 \%$ Seafood compost & $100.0 \mathrm{a}$ & 0 & $3.33 \mathrm{f}-\mathrm{h}$ & 0 & $100.0 \mathrm{a}$ & 0 & $2.20 \mathrm{a}$ & 0.0553 \\
\hline Sunflower & $5 \%$ Willow & $100.0 \mathrm{a}$ & 0 & $3.33 \mathrm{f}-\mathrm{h}$ & 0 & $100.0 \mathrm{a}$ & 0 & $1.55 \mathrm{a}-\mathrm{f}$ & 0.1332 \\
\hline Sunflower & $10 \%$ Willow & $100.0 \mathrm{a}$ & 0 & $3.33 \mathrm{f}-\mathrm{h}$ & 0 & $100.0 \mathrm{a}$ & 0 & $1.08 \mathrm{e}-\mathrm{h}$ & 0.0757 \\
\hline
\end{tabular}

The effect of buckwheat HTCPL was not significantly $(\mathrm{P}>0.05)$ different from that of the seafood compost HTCPL but for the reduced lettuce seed germination rate following treatment with the latter. Consistently, 
willow HTCPL reduced seed germination rate (i.e., 0-48\%) of all the plant species except pea and sunflower, which had seed germination rate of between 95 and $100 \%$ irrespective of HTCPL treatment. Thus, all the HTCPLs including the control recorded high seed germination rate for pea and sunflower seeds (i.e., 95-100\%).

The impacts of the different HTCPLs on seed germination index followed similar trend as seed germination rate (Table 2). Overall, the results suggested that the different HTCPLs did not significantly $(\mathrm{P}>0.05)$ influence seed germination potential compared to the control but was significantly $(\mathrm{P}=0.008)$ reduced by willow HTCPL. Interestingly, sunflower seeds treated with 5\% buckwheat, $10 \%$ seafood compost or 5\% and $10 \%$ willow HTCPLs recorded $100 \%$ seed germination potential: while willow HTCPL reduced seed germination potential of all the other plants. Additionally, willow HTCPL significantly ( $\mathrm{P}=0.001)$ reduced seed vigor in kale, lettuce, pac choi and pea compared to control, buckwheat, and seafood compost HTCPLs (Table 4). The 5\% buckwheat HTCPL seemed to have enhanced seed vigor in pac choi and pea while 5\% and $10 \%$ seafood compost HTCPL enhanced seed vigor in lettuce and sunflower, respectively.

The lengths and surface areas of radicle and hypocotyl were differentially influenced by HTCPL treatment as clearly shown in Figure 1 . These seedling growth components were significantly $(\mathrm{P}<0.05)$ reduced following seed treatment with $5 \%$ or $10 \%$ willow HTCPL compared to the other treatments (Table 5). Overall, growth of kale, lettuce and sunflower radicles and hypocotyls were promoted following treatment with seafood compost HTCPL while pea radicle and hypocotyl growth were promoted following treatment with 5\% buckwheat or $10 \%$ seafood compost HTCPL. The different HTCPLs seemed to have similar effect on pac choi seeds except for willow which had a significant $(\mathrm{P}<0.05)$ reduction effect on all the seedlings in the present study.

Table 5. Length and surface areas of seedling radicles and hypocotyls of kale (Brassica oleracea var. sabellica), lettuce (Lactuca sativa), pac choi (Brassica rapa subsp. chinensis), pea (Pisum sativum) and sunflower (Helianthus annuus) as affected by different sources and high rate of hydrothermal carbonization processed liquid (HTCPL) (different alphabetical letters indicate significant differences between the treatments using 3-way ANOVA and following Tukey's test at $\mathrm{P} \leq 0.05$, confidence intervals $=95 \%$ )

\begin{tabular}{|c|c|c|c|c|c|c|c|c|c|}
\hline \multirow[b]{2}{*}{ Plant } & \multirow[b]{2}{*}{ HTCPL treatments } & \multicolumn{2}{|c|}{ Radicle length } & \multicolumn{2}{|c|}{ Hypocotyl length } & \multicolumn{2}{|c|}{ Radicle surface area } & \multicolumn{2}{|c|}{ Hypocotyl surface area } \\
\hline & & $(\mathrm{cm})$ & sd & $(\mathrm{cm})$ & sd & $(\mathrm{cm} 2)$ & $\mathrm{sd}$ & $(\mathrm{cm} 2)$ & sd \\
\hline Kale & Control & $47.4 \mathrm{abc}$ & 8.64 & $20.6 \mathrm{abc}$ & 6.06 & $8.0 \mathrm{a}-\mathrm{f}$ & 0.697 & $4.4 \mathrm{def}$ & 0.544 \\
\hline Kale & $5 \%$ Buckwheat & $38.1 \mathrm{a}-\mathrm{g}$ & 13.53 & $17.2 \mathrm{~b}-\mathrm{e}$ & 4.82 & $7.2 \mathrm{a}-\mathrm{g}$ & 1.501 & $4.1 \mathrm{e}-\mathrm{h}$ & 0.591 \\
\hline Kale & $10 \%$ Buckwheat & $30.4 \mathrm{~d}-\mathrm{i}$ & 7.86 & $17.1 \mathrm{~b}-\mathrm{e}$ & 5.94 & $6.3 \mathrm{c}-\mathrm{i}$ & 0.885 & $3.9 \mathrm{fgh}$ & 0.647 \\
\hline Kale & $5 \%$ Seafood compost & $49.0 \mathrm{ab}$ & 7.51 & $19.2 \mathrm{bcd}$ & 3.24 & $8.1 \mathrm{a}-\mathrm{e}$ & 1.039 & $3.5 \mathrm{f}-\mathrm{i}$ & 0.472 \\
\hline Kale & $10 \%$ Seafood compost & $39.5 \mathrm{a}-\mathrm{f}$ & 7.78 & $15.2 \mathrm{~b}-\mathrm{f}$ & 3.53 & $7.5 \mathrm{a}-\mathrm{g}$ & 0.878 & $7.5 \mathrm{ab}$ & 0.644 \\
\hline Kale & $5 \%$ Willow & $18.2 \mathrm{i}-\mathrm{m}$ & 6.93 & $7.9 \mathrm{f}-1$ & 2.623 & $4.6 \mathrm{~h}-\mathrm{k}$ & 1.139 & $2.2 \mathrm{jkl}$ & 0.405 \\
\hline Kale & $10 \%$ Willow & $0.7 \mathrm{n}$ & 2.101 & 0.51 & 1.546 & $0.2 \mathrm{~m}$ & 0.546 & $0.2 \mathrm{~m}$ & 0.571 \\
\hline Lettuce & Control & $29.1 \mathrm{e}-\mathrm{j}$ & 6.84 & $11.6 \mathrm{~d}-\mathrm{j}$ & 3.5 & $6.1 \mathrm{~d}-\mathrm{j}$ & 1.078 & $3.2 \mathrm{hi}$ & 0.556 \\
\hline Lettuce & $5 \%$ Buckwheat & 20.9 h-m & 8.17 & $7.4 \mathrm{f}-1$ & 3.14 & $5.1 \mathrm{e}-\mathrm{j}$ & 0.83 & $2.7 \mathrm{ijk}$ & 0.479 \\
\hline Lettuce & $10 \%$ Buckwheat & $20.5 \mathrm{~h}-\mathrm{m}$ & 9.05 & $11.6 \mathrm{~d}-\mathrm{j}$ & 3.86 & $5.6 \mathrm{f}-\mathrm{j}$ & 0.958 & 3.4 ghi & 0.646 \\
\hline Lettuce & $5 \%$ Seafood compost & $46.6 \mathrm{abc}$ & 8.12 & $13.7 \mathrm{~b}-\mathrm{g}$ & 4.47 & $8.5 \mathrm{a}-\mathrm{d}$ & 0.68 & $2.9 \mathrm{~h}-\mathrm{k}$ & 0.598 \\
\hline Lettuce & $10 \%$ Seafood compost & $33.8 \mathrm{~b}-\mathrm{i}$ & 16.24 & $10.7 \mathrm{e}-\mathrm{k}$ & 5.33 & $6.9 \mathrm{a}-\mathrm{i}$ & 2.088 & $6.9 \mathrm{bc}$ & 0.629 \\
\hline Lettuce & $5 \%$ Willow & $7.8 \mathrm{lmn}$ & 2.133 & $6.9 \mathrm{~g}-1$ & 1.339 & $3.1 \mathrm{kj}$ & 0.691 & $2.7 \mathrm{ijk}$ & 0.57 \\
\hline Lettuce & $10 \%$ Willow & $5.1 \mathrm{mn}$ & 1.499 & $4.5 \mathrm{jkl}$ & 0.995 & $2.0 \mathrm{~lm}$ & 0.454 & $2.0 \mathrm{jk}$ & 0.384 \\
\hline Pac choi & Control & $23.2 \mathrm{~g}-1$ & 8.43 & $13.7 \mathrm{~b}-\mathrm{g}$ & 2.159 & $5.9 \mathrm{e}-\mathrm{j}$ & 1.315 & $3.4 \mathrm{ghi}$ & 0.531 \\
\hline Pac choi & $5 \%$ Buckwheat & $23.1 \mathrm{~g}-1$ & 5.08 & $13.3 \mathrm{~b}-\mathrm{g}$ & 2.876 & $5.2 \mathrm{~g}-\mathrm{k}$ & 1.049 & $3.5 \mathrm{f}-\mathrm{i}$ & 0.362 \\
\hline Pac choi & $10 \%$ Buckwheat & $11.9 \mathrm{k}-\mathrm{n}$ & 4.09 & $10.0 \mathrm{e}-\mathrm{k}$ & 2.702 & $3.7 \mathrm{jkl}$ & 0.566 & $3.6 \mathrm{f}-\mathrm{i}$ & 0.395 \\
\hline Pac choi & $5 \%$ Seafood compost & $21.1 \mathrm{~h}-\mathrm{m}$ & 7.92 & $12.4 \mathrm{~d}-\mathrm{i}$ & 4.24 & $5.2 \mathrm{~g}-\mathrm{k}$ & 1.226 & $3.6 \mathrm{f}-\mathrm{i}$ & 0.533 \\
\hline Pac choi & $10 \%$ Seafood compost & $22.9 \mathrm{~g}-1$ & 4.75 & $13.1 \mathrm{~b}-\mathrm{h}$ & 4.03 & & 1.085 & $5.9 \mathrm{c}$ & 0.2832 \\
\hline Pac choi & $5 \%$ Willow & $5.2 \mathrm{mn}$ & 5.5 & $9.0 \mathrm{f}-\mathrm{k}$ & 8.8 & $1.8 \mathrm{~lm}$ & 1.871 & $1.9 \mathrm{jk}$ & 1.805 \\
\hline Pac choi & $10 \%$ Willow & $0.3 n$ & 0.867 & 0.41 & 1.341 & $0.1 \mathrm{~m}$ & 0.406 & $0.2 \mathrm{~m}$ & 0.533 \\
\hline Pea & Control & $27.5 \mathrm{f}-\mathrm{j}$ & 12.23 & $4.2 \mathrm{kl}$ & 2.412 & $6.9 \mathrm{a}-\mathrm{i}$ & 1.92 & $2.6 \mathrm{ijk}$ & 0.769 \\
\hline Pea & $5 \%$ Buckwheat & $41.5 \mathrm{a}-\mathrm{e}$ & 3.57 & $9.2 \mathrm{f}-\mathrm{k}$ & 4.53 & $8.6 \mathrm{ab}$ & 0.938 & $4.1 \mathrm{e}-\mathrm{h}$ & 1.195 \\
\hline Pea & $10 \%$ Buckwheat & $28.0 \mathrm{e}-\mathrm{j}$ & 7.55 & $5.4 \mathrm{i}-1$ & 1.743 & 7.9 a-e & 1.32 & $3.9 \mathrm{fgh}$ & 0.541 \\
\hline Pea & $5 \%$ Seafood compost & $39.2 \mathrm{a}-\mathrm{f}$ & 7.16 & $7.6 \mathrm{~g}-1$ & 2.148 & $8.4 \mathrm{abc}$ & 1.351 & $5.1 \mathrm{cde}$ & 1.014 \\
\hline Pea & $10 \%$ Seafood compost & $34.9 \mathrm{~b}-\mathrm{h}$ & 6.76 & $13.5 \mathrm{c}-\mathrm{g}$ & 8.12 & $8.5 a b c$ & 1.529 & $8.5 \mathrm{a}$ & 1.656 \\
\hline Pea & $5 \%$ Willow & $23.5 \mathrm{~g}-\mathrm{k}$ & 9.22 & $5.9 \mathrm{~h}-1$ & 4.2 & $7.0 \mathrm{a}-\mathrm{g}$ & 0.741 & $2.9 \mathrm{~h}-\mathrm{k}$ & 1.332 \\
\hline Pea & $10 \%$ Willow & $15.2 \mathrm{j}-\mathrm{n}$ & 8.09 & 0.91 & 0.638 & $4.6 \mathrm{hjk}$ & 1.209 & $1.3 \mathrm{kl}$ & 0.522 \\
\hline Sunflower & Control & $39.7 \mathrm{a}-\mathrm{f}$ & 10.87 & $10.7 \mathrm{e}-\mathrm{k}$ & 3.67 & $8.5 \mathrm{abc}$ & 1.626 & $4.3 \mathrm{efg}$ & 1.046 \\
\hline Sunflower & $5 \%$ Buckwheat & $44.9 \mathrm{a}-\mathrm{d}$ & 6.01 & $12.1 \mathrm{~d}-\mathrm{i}$ & 5.34 & $8.9 \mathrm{a}$ & 0.778 & $4.5 \mathrm{def}$ & 1.699 \\
\hline Sunflower & $10 \%$ Buckwheat & $32.9 \mathrm{c}-\mathrm{i}$ & 18.96 & $11.2 \mathrm{e}-\mathrm{j}$ & 7 & $6.8 \mathrm{a}-\mathrm{i}$ & 3.074 & $5.4 \mathrm{~cd}$ & 1.788 \\
\hline Sunflower & $5 \%$ Seafood compost & $49.1 \mathrm{a}$ & 5.5 & $20.4 \mathrm{ab}$ & 6.26 & $8.4 \mathrm{a}-\mathrm{d}$ & 1.576 & $5.9 \mathrm{c}$ & 0.664 \\
\hline Sunflower & $10 \%$ Seafood compost & $46.3 \mathrm{abc}$ & 5.66 & $27.0 \mathrm{a}$ & 5.24 & $6.7 \mathrm{~b}-\mathrm{i}$ & 0.573 & $6.7 \mathrm{bc}$ & 1.281 \\
\hline Sunflower & $5 \%$ Willow & $41.5 \mathrm{a}-\mathrm{e}$ & 4.91 & $10.0 \mathrm{e}-\mathrm{k}$ & 3.074 & $8.7 \mathrm{ab}$ & 0.641 & $5.0 \mathrm{cde}$ & 0.76 \\
\hline Sunflower & $10 \%$ Willow & $22.4 \mathrm{~h}-1$ & 4.41 & $13.5 \mathrm{c}-\mathrm{g}$ & 4.55 & $5.9 e-j$ & 0.891 & $5.0 \mathrm{cde}$ & 0.788 \\
\hline
\end{tabular}




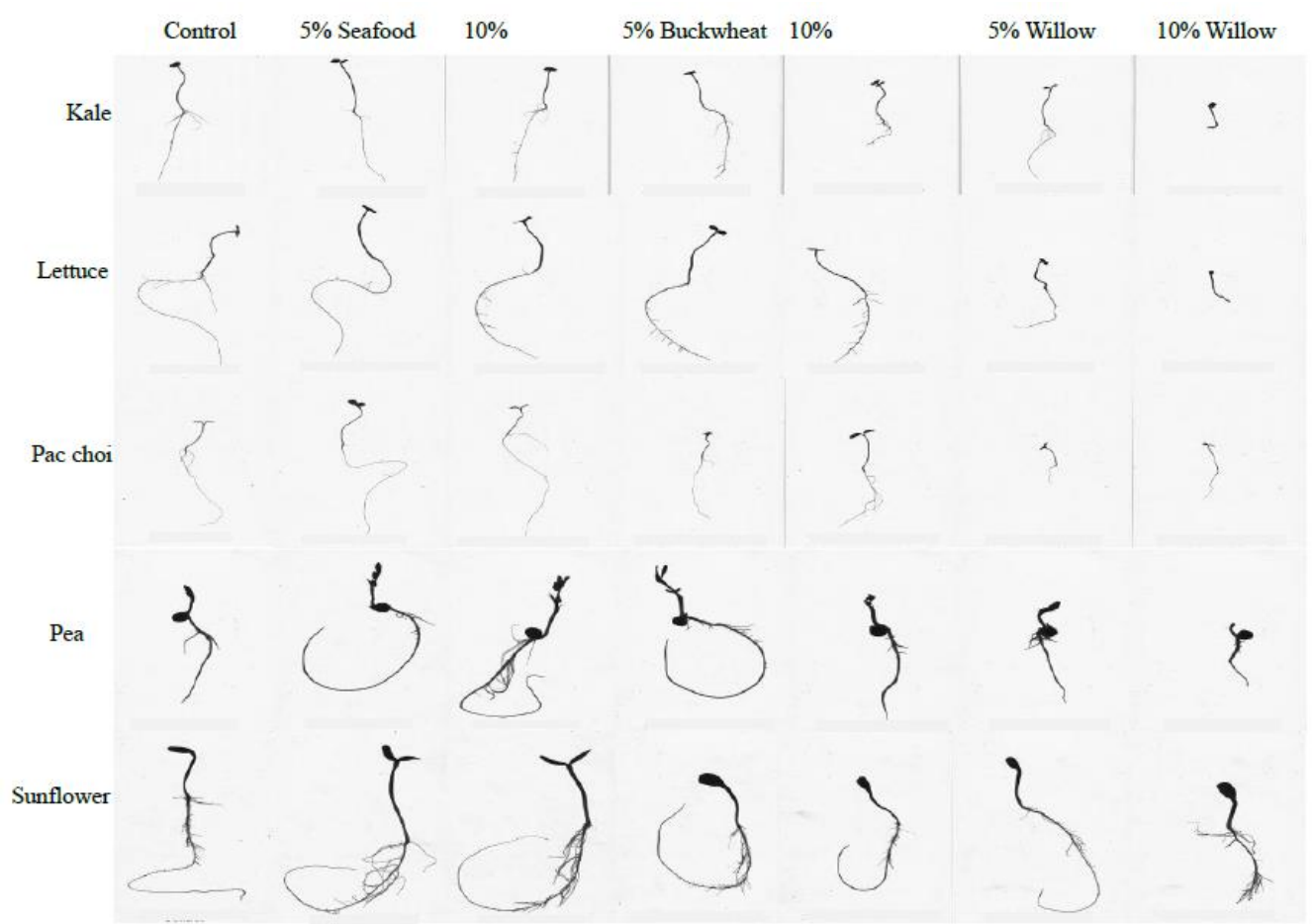

Figure 1. Digital images of seedlings showing differences in size of kale (Brassica oleracea var. sabellica), lettuce (Lactuca sativa), pac choi (Brassica rapa subsp. chinensis), pea (Pisum sativum), and sunflower (Helianthus аппииs) as affected by different sources and high rate of hydrothermal carbonization processed liquid (HTCPL)

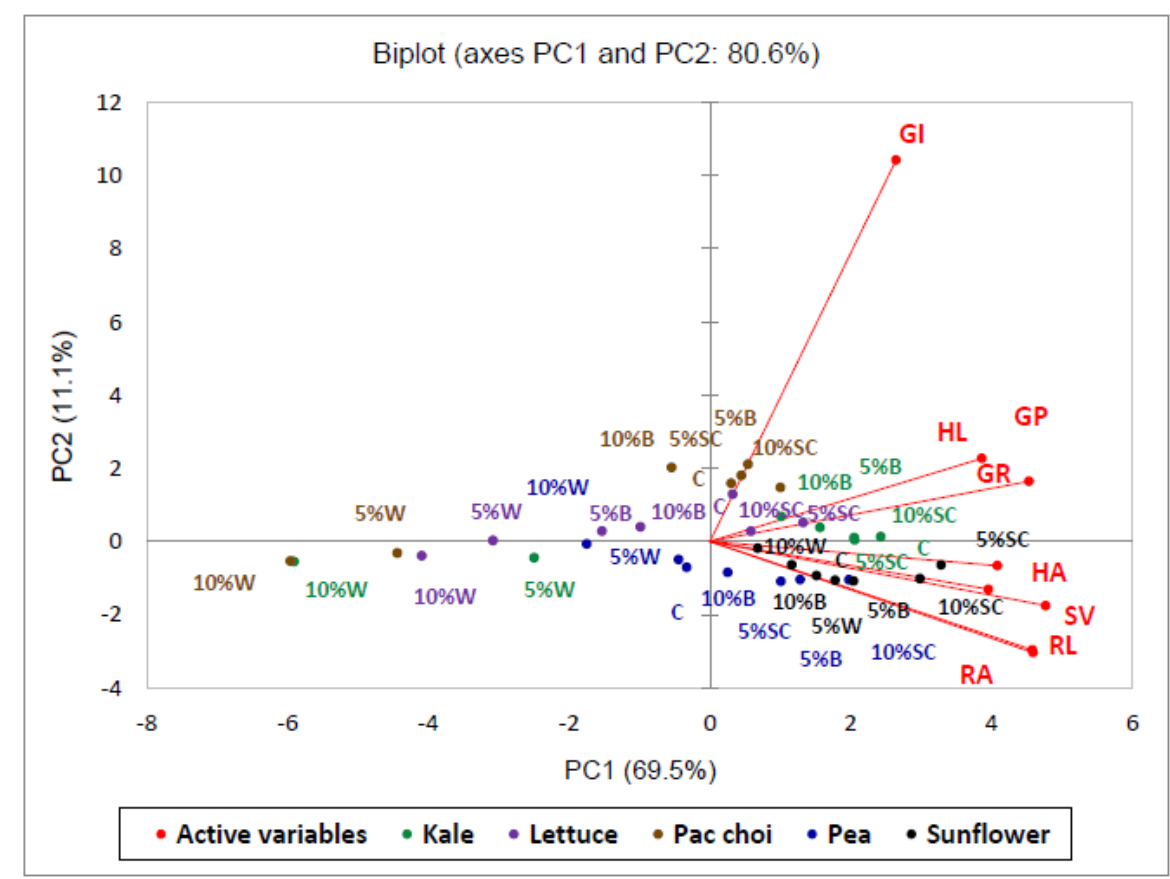

Figure 2. A two-dimensional principal component analysis biplot showing relationships amongst different sources and high rates of application of hydrothermal carbonization processed liquid (HTCPL) and seed germination and seedling growth components of kale (Brassica oleracea var. sabellica), lettuce (Lactuca sativa), pac choi (Brassica rapa subsp. chinensis), pea (Pisum sativum), and sunflower (Helianthus annuus). C, control; W, willow; B, buckwheat; and SC, seafood compost HTCPLs. GR, germination rate; GI, germination index; GP, germination potential; SV, seed vigor index; HL, hypocotyl length; RL, radicle length; HA, hypocotyl surface area; and RA, radicle surface area 
A 2-D PCA biplot demonstrated associations amongst the different HTCPL treatments and responses of the different microgreen plant species (Figure 2). The PCA accounted for $80.6 \%$ of the total variability in dataset. The variations in plant germination and seedling growth components in response to different sources and application rate of HTCPL were clearly shown in the PCA biplot. In general, the impact of HTCPL treatment on seed germination and seedling growth of sunflower was minimal compared to the other plant species. The 5\% and $10 \%$ willow HTCPL treated plants except sunflower had lower level of seed germination rate, seed germination index, seed germination potential, seed vigor index, and reduced hypocotyl length, radicle length, hypocotyl surface area, and radicle surface area (Figure 2). In addition, the buckwheat and seafood compost HTCPL treated plants did not affect seed germination and seedling growth compared to the control treatments.

\section{Experiment 2: Low Application Rate of HTCPL}

There was no significant $(\mathrm{P}>0.05)$ impact of the main effects (HTCPL type or rate) but the 2-way interaction effect were significant $(\mathrm{P}<0.05)$. As such data for the 2-way interaction was presented in Tables 6 and 7 . The results of Experiment 1 demonstrated that the smaller seeds i.e., kale, lettuce and pac choi were more responsive to the HTCPLs compared to the larger seeds i.e., pea and sunflower. In general, the effects of low application rates of willow and buckwheat HTCPLs on kale seed germination rate were comparable except for the $2.5 \%$ willow HTCPL, which had inhibition effect at $40 \%$ germination rate (Table 6).

HTCPL effect on seed germination rate was non-significantly higher for buckwheat HTCPL (i.e., average of 98\%) compared to willow HTCPL (i.e., average of 94\%) with the control at 95\%. Furthermore, control, $0.5 \%$ of willow HTCPL and $0.5 \%$ or $2.5 \%$ buckwheat HTCPL consistently gave the highest seed germination potential, seed germination index and seed vigor (Table 6). It was obvious that variation in the application rate of buckwheat HTCPL application did not alter seed germination, germination potential, germination index and seed vigor index. Like the seed germination rate, seed vigor was also reduced by $2.5 \%$ willow HTCPL compared to the other treatments. On average, willow HTCPL significantly $(\mathrm{P}<0.05)$ reduced kale seed vigor and seed germination components, while the control and buckwheat HTCPL had incremental effect across the different application rates.

Table 6. Seed germination indices of kale (Brassica oleracea var. sabellica) as affected by different sources and low rate of hydrothermal carbonization processed liquid (HTCPL) (different alphabetical letters indicate significant differences between the treatments using 2-way ANOVA and following Tukey's test at $\mathrm{P} \leq 0.05$, confidence intervals $=95 \%$ )

\begin{tabular}{lllllllll}
\hline & \multicolumn{2}{l}{ Germination rate } & \multicolumn{2}{l}{ Germination potential } & \multicolumn{2}{l}{ Germination index } & \multicolumn{2}{l}{ Seed vigor index } \\
\hline Treatment & $(\%)$ & $\mathrm{sd}$ & $(\%)$ & $\mathrm{sd}$ & $/$ & $\mathrm{sd}$ & $/$ & $\mathrm{sd}$ \\
\hline Control & $95.0 \mathrm{a}$ & 0.1 & $90.0 \mathrm{a}$ & 0.1155 & $9.188 \mathrm{a}$ & 0.987 & $0.512 \mathrm{a}$ & 0.0519 \\
0.5\% Willow HTCPL & $92.5 \mathrm{a}$ & 0.0957 & $77.5 \mathrm{a}$ & 0.1258 & $8.313 \mathrm{ab}$ & 0.862 & $0.522 \mathrm{a}$ & 0.0675 \\
$1 \%$ Willow HTCPL & $95.0 \mathrm{a}$ & 0.1 & $55.0 \mathrm{~b}$ & 0.1 & $7.008 \mathrm{~b}$ & 0.995 & $0.525 \mathrm{a}$ & 0.0972 \\
$2.5 \%$ Willow HTCPL & $40.0 \mathrm{~b}$ & 0.271 & $25.0 \mathrm{c}$ & 0.1 & $2.342 \mathrm{c}$ & 0.236 & $0.172 \mathrm{~b}$ & 0.1202 \\
0.5\% Buckwheat HTCPL & $97.5 \mathrm{a}$ & 0.05 & $90.0 \mathrm{a}$ & 0 & $9.300 \mathrm{a}$ & 0.245 & $0.513 \mathrm{a}$ & 0.0943 \\
$1 \%$ Buckwheat HTCPL & $97.5 \mathrm{a}$ & 0.05 & $97.5 \mathrm{a}$ & 0.05 & $9.750 \mathrm{a}$ & 0.5 & $0.453 \mathrm{a}$ & 0.0321 \\
$2.5 \%$ Buckwheat HTCPL & $97.5 \mathrm{a}$ & 0.05 & $85.0 \mathrm{a}$ & 0.0577 & $9.125 \mathrm{a}$ & 0.25 & $0.455 \mathrm{a}$ & 0.0233 \\
\hline
\end{tabular}

Radicle length of kale seedlings was increased following treatment with $0.5 \%$ willow HTCPL but was not significantly $(\mathrm{P}>0.05)$ different from the control and the other treatments. However, $2.5 \%$ buckwheat HTCPL significantly $(\mathrm{P}=0.001)$ reduced radicle length (Table 7$)$. The highest seedling hypocotyl length was recorded by $1 \%$ willow HTCPL and was also significantly $(\mathrm{P}=0.001)$ higher than that for the $2.5 \%$ willow HTCPL.

Again, $0.5 \%$ willow HTCPL highly increased the surface area of kale seedling radicles while $1 \%$ willow and $0.5 \%$ buckwheat HTCPLs highly increased the surface area of hypocotyls (Table 7). Most of the treatments had similar impacts on seedling growth components. Reductions in seedling growth components was not consistent with treatment. For example, 2.5\% buckwheat HTCPL gave the least radicle length and radicle surface area. The $2.5 \%$ willow HTCPL gave the least hypocotyl length and the $2.5 \%$ willow HTCPL gave the least hypocotyl surface area (Table 7). On average, the control followed by willow HTCPL was superior for radicle length and radicle surface area while buckwheat HTCPL was superior for hypocotyl length and hypocotyl surface area. 
Table 7. Length and surface areas of seedling radicles and hypocotyls of kale (Brassica oleracea var. sabellica) as affected by different sources and low rate of hydrothermal carbonization processed liquid (HTCPL) (different alphabetical letters indicate significant differences between the treatments using 2-way ANOVA and following Tukey's test at $\mathrm{P} \leq 0.05$, confidence intervals $=95 \%$ )

\begin{tabular}{lllllllll}
\hline & \multicolumn{3}{l}{ Radicle length } & \multicolumn{2}{l}{ Hypocotyl length } & \multicolumn{2}{l}{ Radicle surface area } & \multicolumn{2}{l}{ Hypocotyl surface area } \\
\hline Treatment & $(\mathrm{cm})$ & $\mathrm{sd}$ & $(\mathrm{cm})$ & $\mathrm{sd}$ & $(\mathrm{cm} 2)$ & $\mathrm{sd}$ & $(\mathrm{cm} 2)$ & $\mathrm{sd}$ \\
\hline Control & $31.241 \mathrm{ab}$ & 2.12 & $22.645 \mathrm{ab}$ & 1.425 & $5.525 \mathrm{ab}$ & 0.266 & $5.658 \mathrm{ab}$ & 0.303 \\
0.5\% Willow HTCPL & $33.784 \mathrm{a}$ & 3.31 & $22.749 \mathrm{ab}$ & 2.17 & $6.160 \mathrm{a}$ & 0.547 & $5.422 \mathrm{ab}$ & 0.209 \\
1\% Willow HTCPL & $27.100 \mathrm{abc}$ & 3.18 & $27.992 \mathrm{a}$ & 5.09 & $5.324 \mathrm{ab}$ & 0.1142 & $6.451 \mathrm{a}$ & 0.594 \\
2.5\% Willow HTCPL & $24.736 \mathrm{abc}$ & 6.06 & $18.611 \mathrm{~b}$ & 6.07 & $4.759 \mathrm{ab}$ & 1.252 & $4.712 \mathrm{~b}$ & 0.665 \\
0.5\% Buckwheat HTCPL & $25.954 \mathrm{abc}$ & 6.41 & $26.514 \mathrm{ab}$ & 2.4 & $4.826 \mathrm{ab}$ & 1.19 & $6.448 \mathrm{a}$ & 0.521 \\
$1 \%$ Buckwheat HTCPL & $22.225 \mathrm{bc}$ & 3.67 & $24.191 \mathrm{ab}$ & 4.03 & $4.988 \mathrm{ab}$ & 0.729 & $6.052 \mathrm{ab}$ & 1.064 \\
2.5\% Buckwheat HTCPL & $20.964 \mathrm{c}$ & 1.457 & $25.670 \mathrm{ab}$ & 2.09 & $4.308 \mathrm{~b}$ & 0.502 & $6.000 \mathrm{ab}$ & 0.451 \\
\hline
\end{tabular}

A 2-D PCA biplot explained $89.2 \%$ of the total variability in dataset (Figure 3). Obviously, the kale treated with $2.5 \%$ willow HTCPL had low level of seed germination and seedling growth components (Figure 3). The $0.5 \%$ willow HTCPL and the control treated kales had highest radicle length and radicle surface area but lowest hypocotyl length and hypocotyl surface area (Figure 3). Conversely, buckwheat HTCPL reduced kale seedling radicle length and radicle surface area but increased hypocotyl length and hypocotyl surface area (Figure 3). Likewise, kale seed germination rate, seed germination index and seed germination potential were increased by $0.5 \%$ buckwheat HTCPL. The $1 \%$ willow HTCPL also increased kale seed germination rate, seed vigor index and hypocotyl surface area.

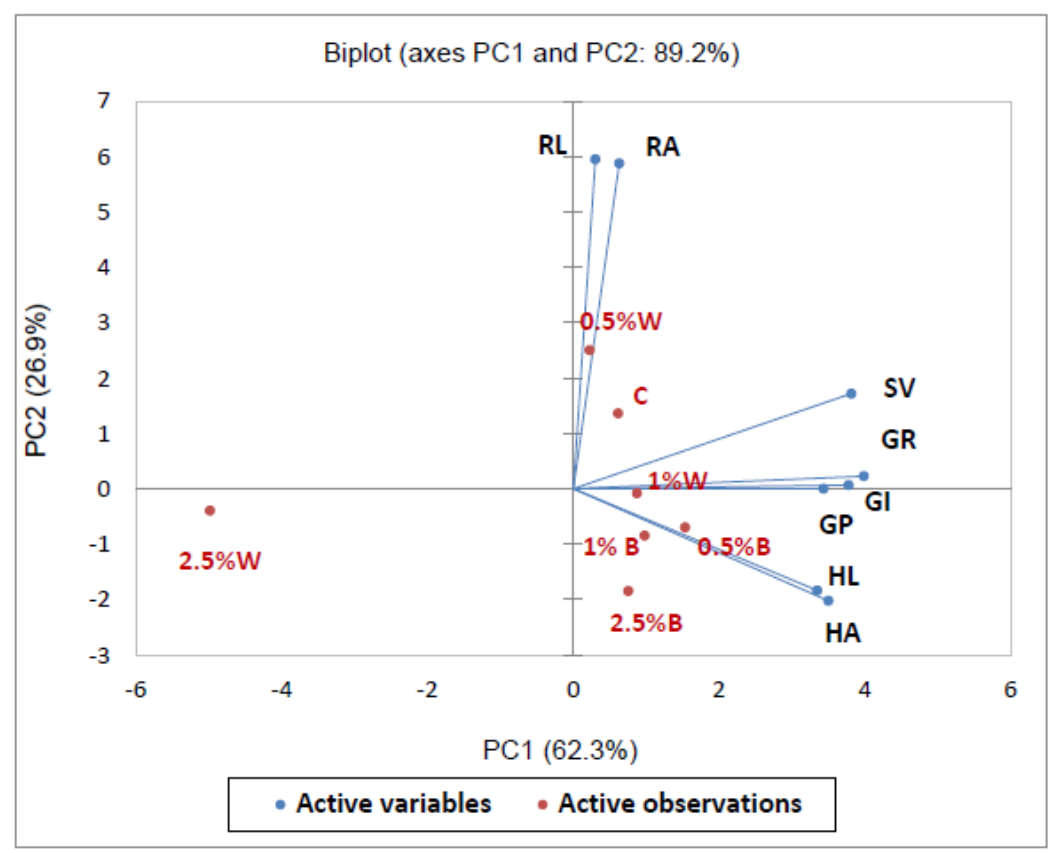

Figure 3. A two-dimensional principal component analysis biplot showing relationships amongst different sources and low rates of application of hydrothermal carbonization processed liquid (HTCPL) and seed germination and seedling growth components of kale (Brassica oleracea var. sabellica). C, control; W, willow; B, buckwheat; and SC, seafood compost HTCPLs. GR, germination rate; GI, germination index; GP, germination potential; SV, seed vigor index; HL, hypocotyl length; RL, radicle length; HA, hypocotyl surface area; RA, radicle surface area

\section{Discussion}

The change in concentrations of individual elements seemed to be dependent on the type of feedstock and the nature and form of compounds from which the elements were made available in the HTCPL. These can explain the controversy in the literature regarding the different outcomes on HTCPL elemental with reference to the starting feedstock (Heilmann et al., 2010; Funke et al., 2013; Funke, 2015; Idowu et al., 2017). Compared to seafood HTCPL and willow HTCPL, the buckwheat HTCPL retained more N. Exposure of plant biomass to high temperature and pressure causes cellular disintegration and decomposition of complex chemical compounds such 
as lignin, cellulose, hemicellulose, proteins and lipids into simpler organic compounds and chemical elements (Drygaś, Depciuch, Puchalski, \& Zaguła, 2016). It seemed the high temperature (i.e., $200^{\circ} \mathrm{C}$ ) and pressure (i.e., $100 \mathrm{psi}$ ) of the HTC process caused volatilization of some chemicals such as $\mathrm{N}$ and allied compounds, which led to their reductions in the extracted HTCPLs. Compost mostly contains organic $\mathrm{N}$ which might have been adversely affected by the intense heat during the HTC process.

Willow is high in organic acids, particularly acetic acid and other carboxylic acids (Hager et al., 2020). This explains the low willow HTCPL pH of 3.7 compared to buckwheat HTCPL pH of 4.6 and seafood compost HTCPL $\mathrm{pH}$ of 5.1. Seed germination requires a pH >5.5 (Roem, Klees, \& Berendse, 2002; Koger, Reddy, \& Poston, 2004). The pH values for the 5\% and 10\% willow HTCPLs were 4.04 and 3.75 respectively, which may be outside the desirable $\mathrm{pH}$ limit for the seed germination. After germination, most plants grow well at $\mathrm{pH}$ between 5 - 8 (Liu and Hanlon, 2015) and therefore, dilution of the willow and buckwheat HTCPLs before application is necessary for most plants. Alkalinity determines carbonate and bicarbonate levels and can directly affect $\mathrm{pH}$. The range of alkalinity suitable for most plant growth is 0 to $160 \mathrm{mg} / \mathrm{kg}$ (Roosta, 2011). As a result, the high alkalinity of $961.10 \mathrm{mg} / \mathrm{kg}$ for buckwheat HTCPL, if not diluted, can impair plant growth and development. The alkalinity of seafood compost and willow HTCPLs were 351.25 and $97.6 \mathrm{mg} / \mathrm{kg}$, respectively. Overall, the elemental composition of the seafood compost, buckwheat, and willow HTCPLs demonstrated potential use as fertilizers.

Experiment 1 demonstrated that the relatively large sunflower and pea seeds were less sensitive to high rate (i.e., 5-10\%) of HTCPLs compared to the smaller seeds of kale, lettuce and pac choi seeds. Variations in plant genotypic characteristics as well as seed size and other physical characteristics might have played a pivotal role in the differential plant responses to HTCPLs as previously explained by researchers (Hegarty, 1976; Fenner and Lee, 1989; Hanley and Fenner, 1997; Akinyosoye, Adetumbi, Amusa, Olowolafe, \& Olasoji, 2014). It is therefore, suggested that the larger seeds of sunflower and pea were less impacted by the HTCPLs by virtue of available food and energy reserves, and activities of enzymes and proteins (Rynd, 1978; Özgen, Yildiz, Koyuncu, \& Önde, 2007).

The impacts of HTCPLs elemental composition, $\mathrm{pH}$, and alkalinity were significant. However, it seemed the high acidity in the willow HTCPL rather than elemental composition was the main cause of inhibition of seed germination and reduction in seedling growth. These findings can be further explained by the PCA biplots. Locations of the different willow HTCPL application rates compared to those of the other HTCPLs in the PCA biplot confirmed inhibition effects of the former.

The rational for Experiment 2 was to focus the study on low rates $(0-2.5 \%)$ of buckwheat and willow HTCPLs application on germination indices of small seeds i.e., kale. Low rates of the HTCPLs did not significantly (P>0.05) influence kale seed germination. With reference to the control, high rates of HTCPLs application in Experiment 1 appeared to have clear advantage on seedling growth unlike the low rates in Experiment 2. Hence, the similarities in effect of the different sources of HTCPLs on seed vigor and seed germination components in Experiment 2 except for $2.5 \%$ willow HTCPL that reduced seed germination and seedling growth. Willow HTCPL at $0.5 \%$ can be positively associated with radicle length and radicle surface area; while the other HTCPL treatments except for $2.5 \%$ willow HTCPL can be associated with all the other seed germination and seedling growth components. Previous studies have shown that HTCPLs are rich in polyphenols (e.g., tannins) and volatile fatty acids that can inhibit seed germination and seedling growth (Puccini et al., 2018). It is therefore, surmised that the presence of appreciable amounts of polyphenols and volatile fatty acids in addition to low $\mathrm{pH}$ in some of the HTCPL treatments may be the reason for the inhibition of seeds and seedling growth in the present study.

\section{Conclusion}

It is concluded that HTCPL has potential for use as seed germination and seedling growth promoter. However, HTCPL from different sources of biomass have varied $\mathrm{pH}$ and chemical composition with different impact on seed germination and seedling growth. The effect of HTCPL is thus dependent on plant species, type of feedstock and application rate. Significant impact of HTCPL can be realized at higher application rate of between 5 and $10 \%$ depending on the biomass. Typically, willow HTCPL is very acidic, and can have negative impact on seed germination and seedling growth. Consistently, buckwheat and seafood compost HTCPLs at high application rates of $5 \%$ and $10 \%$ proved to be more effective compared to willow HTCPL. At low application rate such as $0.5 \%$ and $1 \%$, buckwheat HTCPL seemed to be superior. Until now, there are only few studies explicitly evaluating the effect of HTCPL on seed germination and seedling growth. As such, further investigation will be required to confirm the present results and efficacy of HTCPL obtained from a wide range 
of biomass extracted at different HTC process temperature and pressure regimes. A comprehensive non-targeted chemical profile of HTCPL will also help to explain mechanisms.

\section{Funding}

This work was not supported by any external funding.

\section{Acknowledgment}

The lead author, Yuhang He, wishes to thank Dr. Samuel K. Asiedu and Dr. Lokanadha R. Gunupuru and all her lab mates for their support and suggestions during the study.

\section{References}

Abbey, L., Annan, N., Asiedu, S., Esan, E., \& Iheshiulo, E. (2018). Amino Acids, Mineral Nutrients, and Efficacy of Vermicompost and Seafood and Municipal Solid Wastes Composts. International Journal of Agronomy, 2018, 1-6. https://doi.org/10.1155/2018/6419467

Akinyosoye, S. T., Adetumbi, J. A., Amusa, O. D., Olowolafe, M. O., \& Olasoji, J. O. (2014). Effect of seed size on in vitro seed germination, seedling growth, embryogenic callus induction and plantlet regeneration from embryo of maize (Zea mays L.) seed. Nigerian Journal of Genetics, 28(2), 1-7. https://doi.org/10.1016/j.nigjg.2015.06.001

Bargmann, I., Rillig, M. C., Buss, W., Kruse, A., \& Kuecke, M. (2013). Hydrochar and biochar effects on germination of spring barley. Journal of agronomy and crop science, 199(5), 360-373. https://doi.org/10.1111/jac.12024

Cataldo, D. A., Schrader, L. E., \& Youngs, V. L. (1974). Analysis by Digestion and Colorimetric Assay of Total Nitrogen in Plant Tissues High in Nitrate 1. Crop Science, 14(6), 854-856. https://doi.org/10.2135/cropsci1974.0011183X001400060024x

Ching, T. M., \& Rynd, L. (1978). Developmental differences in embryos of high and low protein wheat seeds during germination. Plant Physiology, 62(6), 866-870. https://doi.org/10.1104/pp.62.6.866

Drygaś, B., Depciuch, J., Puchalski, C., \& Zaguła, G. (2016). The impact of heat treatment on the components of plant biomass as exemplified by Junniperus sabina and Picea abies. ECONTECHMOD: An International Quarterly Journal on Economics of Technology and Modelling Processes, 5. Retrieved from http://yadda.icm.edu.pl/baztech/element/bwmeta1.element.baztech-384e0797-c4fb-412b-8d2b-5b1b267233 d2/c/Drygas_B.pdf

Escala, M., Zumbuhl, T., Koller, C., Junge, R., \& Krebs, R. (2013). Hydrothermal carbonization as an energy-efficient alternative to established drying technologies for sewage sludge: a feasibility study on a laboratory scale. Energy \& Fuels, 27(1), 454-460. https://doi.org/10.1021/ef3015266

Funke, A. (2015). Fate of plant available nutrients during hydrothermal carbonization of digestate. Chemie Ingenieur Technik, 87(12), 1713-1719. https://doi.org/10.1002/cite.201400182

Funke, A., Mumme, J., Koon, M., \& Diakité, M. (2013). Cascaded production of biogas and hydrochar from wheat straw: Energetic potential and recovery of carbon and plant nutrients. Biomass and bioenergy, 58, 229-237. https://doi.org/10.1016/j.biombioe.2013.08.018

Hagner, M., Tiilikkala, K., Lindqvist, I., Niemelä, K., Wikberg, H., Källi, A., \& Rasa, K. (2020). Performance of liquids from slow pyrolysis and hydrothermal carbonization in plant protection. Waste and Biomass valorization, 11(3), 1005-1016. https://doi.org/10.1007/s12649-018-00545-1

Hanley, M. E., \& Fenner, M. (1997). Seedling growth of four fire-following Mediterranean plant species deprived of single mineral nutrients. Functional Ecology, 11(3), 398-405. https://doi.org/10.1046/j.1365-2435.1997.00104.x

Hegarty, T. W. (1976). Effects of fertilizer on the seedling emergence of vegetable crops. Journal of the Science of Food and Agriculture, 27(10), 962-968. https://doi.org/10.1002/jsfa.2740271014

Heidari, M., Salaudeen, S., Dutta, A., \& Acharya, B. (2018). Effects of process water recycling and particle sizes on hydrothermal carbonization of biomass. Energy \& Fuels, 32(11), 11576-11586. https://doi.org/10.1021/acs.energyfuels.8b02684

Heilmann, S. M., Davis, H. T., Jader, L. R., Lefebvre, P. A., Sadowsky, M. J., Schendel, F. J., ... Valentas, K. J. (2010). Hydrothermal carbonization of microalgae. Biomass and Bioenergy, 34(6), 875-882.

https://doi.org/10.1016/j.biombioe.2010.01.032 
Idowu, I., Li, L., Flora, J. R., Pellechia, P. J., Darko, S. A., Ro, K. S., \& Berge, N. D. (2017). Hydrothermal carbonization of food waste for nutrient recovery and reuse. Waste Management, 69, 480-491. https://doi.org/10.1016/j.wasman.2017.08.051

Koger, C. H., Reddy, K. N., \& Poston, D. H. (2004). Factors affecting seed germination, seedling emergence, and survival of texasweed (Caperonia palustris). Weed science, 52(6), 989-995. https://doi.org/10.1614/WS-03-139R2

Liu, G., \& Hanlon, E. (2012). Soil pH range for optimum commercial vegetable production.

Ma, C., Song, K., Yu, J., Yang, L., Zhao, C., Wang, W., ... \& Zu, Y. (2013). Pyrolysis process and antioxidant activity of pyroligneous acid from Rosmarinus officinalis leaves. Journal of Analytical and Applied Pyrolysis, 104, 38-47. https://doi.org/10.1016/j.jaap.2013.09.011

Özgen, M., Yildiz, M., Koyuncu, N., \& Önde, S. (2007). The effect of seed size on tissue culture response of callus from endosperm-supported mature embryos of barley (Hordeum vulgare L.). Cereal Research Communications, 35(3), 1415-1425. https://doi.org/10.1016/j.biombioe.2012.12.004

Puccini, M., Ceccarini, L., Antichi, D., Seggiani, M., Tavarini, S., Hernandez Latorre, M., \& Vitolo, S. (2018). Hydrothermal. carbonization of municipal woody and herbaceous prunings: Hydrochar valorisation as soil amendment and growth medium for horticulture. Sustainability, 10(3), 846. https://doi.org/10.3390/su10030846

Reza, M. T., Lynam, J. G., Uddin, M. H., \& Coronella, C. J. (2013). Hydrothermal carbonization: Fate of inorganics. Biomass and Bioenergy, 49, 86-94. https://doi.org/10.1016/j.biombioe.2012.12.004

Roem, W. J., Klees, H., \& Berendse, F. (2002). Effects of nutrient addition and acidification on plant species diversity and seed. germination in heathland. Journal of Applied Ecology, 39(6), 937-948. https://doi.org/10.1046/j.1365-2664.2002.00768.x

Roosta, H. R. (2011). Interaction between water alkalinity and nutrient solution $\mathrm{pH}$ on the vegetative growth, chlorophyll fluorescence and leaf magnesium, iron, manganese, and zinc concentrations in lettuce. Journal of Plant Nutrition, 34(5), 717-731. https://doi.org/10.1080/01904167.2011.540687

Saetea, P., \& Tippayawong, N. (2013). Recovery of value-added products from hydrothermal carbonization of sewage sludge. International Scholarly Research Notices, 2013. http://dx.doi.org/10.1155/2013/268947

Salehi, A., Mehdi, B., Fallah, S., Kaul, H. P., \& Neugschwandtner, R. W. (2018). Productivity and nutrient use efficiency with integrated fertilization of buckwheat-fenugreek intercrops. Nutrient cycling in agroecosystems, 110(3), 407-425. https://doi.org/10.1007/s10705-018-9906-x

Sun, X., Sumida, H., \& Yoshikawa, K. (2014). Effects of liquid fertilizer produced from sewage sludge by the hydrothermal process on the growth of Komatsuna. British Journal of Environment and Climate Change, 4(3), 261-278. https://doi.org/10.9734/BJECC/2014/11636

Yao, C., Pan, Y., Lu, H., Wu, P., Meng, Y., Cao, X., \& Xue, S. (2016). Utilization of recovered nitrogen from hydrothermal carbonization process by Arthrospira platensis. Bioresource technology, 212, 26-34. https://doi.org/10.1016/j.biortech.2016.03.166

Yang, R., Guo, L., Jin, X., Shen, C., Zhou, Y., \& Gu, Z. (2015). Enhancement of glucosinolate and sulforaphane formation of broccoli sprouts by zinc sulphate via its stress effect. Journal of Functional Foods, 13, 345-349. https://doi.org/10.1016/j.jff.2015.01.007

Yousefi, S., Kartoolinejad, D., Bahmani, M., \& Naghdi, R. (2017). Effect of Azospirillum lipoferum and Azotobacter chroococcum on germination and early growth of hopbush shrub (Dodonaea viscosa L.) under salinity stress. Journal of Sustainable Forestry, 36(2), 107-120.

https://doi.org/10.1080/10549811.2016.1256220

\section{Copyrights}

Copyright for this article is retained by the author(s), with first publication rights granted to the journal.

This is an open-access article distributed under the terms and conditions of the Creative Commons Attribution license (http://creativecommons.org/licenses/by/3.0/). 